Voix et Images

\title{
Archéologie du littéraire au Québec : bibliographie
}

\section{Isabelle Beaulé}

Volume 20, numéro 2 (59), hiver 1995

Archéologie du littéraire au Québec

URI : https://id.erudit.org/iderudit/201170ar

DOI : https://doi.org/10.7202/201170ar

Aller au sommaire du numéro

\section{Éditeur(s)}

Université du Québec à Montréal

ISSN

0318-9201 (imprimé)

1705-933X (numérique)

Découvrir la revue

Citer cet article

Beaulé, I. (1995). Archéologie du littéraire au Québec : bibliographie. Voix et Images, 20(2), 388-397. https://doi.org/10.7202/201170ar d'utilisation que vous pouvez consulter en ligne.

https://apropos.erudit.org/fr/usagers/politique-dutilisation/ 


\title{
Archéologie du littéraire au Québec: bibliographie
}

\author{
Isabelle Beaulé, Université du Québec à Montréal
}

La présente bibliographie comporte les ouvrages les plus cités dans nos articles et ceux consultés aux fins de ce dossier. Pour une liste plus exhaustive, on consultera principalement la bibliographie de La vie littéraire au Québec (Lemire, 1991, 1992).

\section{CORPUS LITTÉRAIRE}

Textes publiés des auteurs de notre corpus (on se reportera aux articles du dossier pour les écrits demeurant dans des dépôts d'archives, ainsi que pour les références en histoire de l'art). On trouvera à la fin de cette section un certain nombre d'anthologies relatives à nos œuvres, ou des rééditions de celles-ci.

\subsection{Textes}

ANONYME ${ }^{1}$, La Bastille septentrionale, ou les trois sujets britanniques opprimés, Montréal, Fleury Mesplet, \&c., 1791, 32 p.

BABY, François, Le Canadien et sa femme [1794], J. Hare (éd.), Ottawa, édition de l'Université d'Ottawa, "Fontenay", 1994, 30 p.

BADEAUX, Jean-Baptiste, "Journal des opérations de l'armée américaine lors de l'invasion du Canada en 1775-1776 ", La Revue canadiènne, vol. VII, mars 1870 , p. $186-202$; vol. VII, avril 1870 , p. $267-286$; vol. VII, mai 1870 , p. $329-$ 345.

BAILLY DE MESSEIN, Charles-François, Copie de la lettre de l'évêque de Capsa coadjuteur dé Québec, Ec. au président du Comité sur l'éducation, Québec, Samuel Neilson, 1790, [3]-10-10 [1] p.

BEREY, Félix de, "Réplique par le P. de Berey aux calomnies de Pierre du Calvet contre les Recolets de Québec [1784] ", Rapport sur les Archives du Canada, 1888, Ottawa, Brown Chamberlin, 1889, p. 40-43.

CASGRAIN, Henri-Raymond (dir.), Lettres du marquis de Montcalm au chevalier de Lévis - Collection des manuscrits du maréchal de Lévis - tome VI, Québec, L.-J. Demers, 1894, 240 p.

1. Attribué à Henri-Antoine Mézière. 
CUTHBERT, Ross, L'Areopage, Québec, J. Neilson 1803, 13-[1] p.

Considerations, \&c. par un Canadien, M.P.P. ., Québec, J. Neilson 1809 [2]-3[2] $\mathrm{p}$.

DU CALVET, Pierre, Mémoire en réponse à l'écrit public, de Me Panet, Ec., Montréal, Fleury Mesplet, 1779, 25-[1] p.

—, Appel à la Justice de l'État ou Recueil de Lettres au Roi, Ec., Londres, s. éd., 1784, xiv-320-viii p. [voir aussi infra Lagrave et Ruelland, 1986].

DUMAS, Alexandre, Discours prononcé par Mr. Alexandre Dumas au Club constitutionnel, tenu à Québec, le 30 mai 1792. Imprimé pour l'instruction des électeurs de la province du Bas-Canada, \&c., Québec, Samuel Neilson, 1792, 3 p.

Gazette de Québec/Quebec Gazette, 1764-1817, hebdomadaire.

Gazette littéraire pour la ville et district de Montréal, 1778-1779, hebdomadaire.

GRASSET DE SAINT-SAUVEUR, Jacques, Encyclopédie des voyages, contenant l'abrégé bistorique des mours, usages, babitudes domestiques, religions, Ec., Paris, Du Roy, 1796, 5 vol.

HUBERT, J.-F. et William SMITH, Rapport du comité du conseil, sur l'objet d'augmenter les moiens d'éducation Ec., Quèbec, Samuel Neilson, 1790, [26p.].

HUET DE LA VALINIÈRE, Pierre, Vraie bistoire ou simple Précis des infortunes pour ne pas dire, des Persécutions qu'a souffert et souffre encore le Révérend Pierre Huet de la Valinière, Mis en vers par lui-même en juillet 1792, à Albany, Ch. et G. Webster, 1792, 50 p.

JAUTARD, Valentin, [ voir infra Lagrave et Ruelland, 1989 et Gazette littéraire pour la ville et district de Montréal.

LA CORNE, dit LA CORNE SAINT-LUC, Luc de, Journal du voyage de M. Saint-Luc de La Corne, ecuyer, dans le navire l'Auguste, en l'an 1761, Montréal, Fleury Mesplet, 1778, 38 p. [voir infra John Hare, pour l'édition critique].

MÉZIÈRE, Henri-Antoine, "Un mémoire de Henri Mézière ", Bulletin des recherches bistoriques, vol. XXXVII, $\mathrm{n}^{\circ}$ 4, avril 1931, p. 193-201.

PATOUILLET, l'abbé (éd.), "Lettre du P. Roubaud, missionnaire chez les Abnakis ", Lettres édifiantes et curieuses écrites des missions étrangères par quelques missionnaires de la $C$. de J., Paris, Chez Charles Pierre Berton, XXXIII recueil, 1776 , p. 210- 297.

PLAMONDON, Louis, Almanach des dames, pour l'année 1807, par un jeune Canadien, Québec, Nouvelle Imprimerie, [1806?], 64 p.

Séance de la Société littéraire de Québec, tenue samedi, le 3e juin, 1809, Québec, J. Neilson, 1809, 21-[1] p.

PLESSIS, Joseph-Octave, Discours à l'occasion de la victoire remportée par les forces navales de Sa Majestée britannique dans.la Méditerrannée le 1 et 2 août 1798, sur la flotte française. Ec., Québec, J. Neilson, 1799, [6]-24 p. 


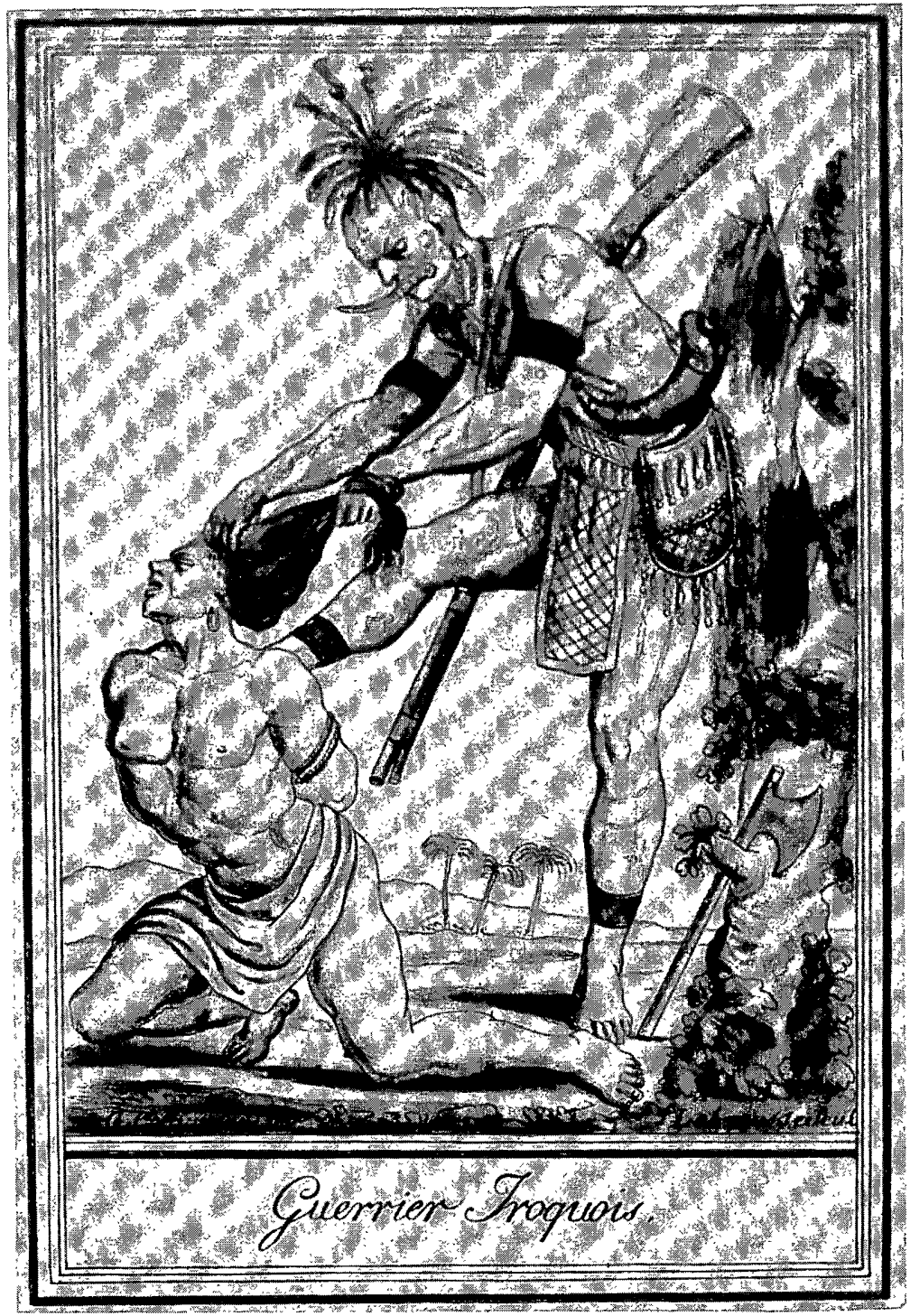

Jacques Grasset de Saint-Sauveur (inv.) et Labrousse (Scul), Guerrier iroquois (Encyclopédie des voyages..., Paris, Du Roy, 1796, vol. V), gravure aquarellée, 17,2 x 11,5 cm, Montréal, musée David M.-Stewart (970-601-1). Un écrivain et illustrateur né à Montréal (1757-1810), dont la carrière s'est déroulée en France au tournant du $\mathrm{xrx}^{\mathrm{e}}$ siècle. Il a produit une vingtaine de volumes et plusieurs centaines d'images d'une grande beauté encore peu étudiées de ce côté-ci de l'Atlantique. Où se situe sa représentation de l'Amérindien parmi celles bien connues des XvII ${ }^{\mathbf{e}}$ et $\mathrm{XIX}^{\mathrm{e}}$ siècles? Est-ce le texte ou l'image qui a assuré le succès de ses publications? 
Journal d'un voyage en Europe par $M^{g r}$ Joseph-Octave Plessis 11819 1820l, Québec, Pruneau \& Kirouac, 1903, 469 p.

, Oraison funèbre de $M^{g r}$ Jean-Olivier Briand, ancien évêque de Québec, prononcée dans la catbédrale de Québec le 27 juin 1794, Lévis, Bulletin des recherches historiques, 1906, $26 \mathrm{p}$.

QUESNEL, Joseph, Colas et Colinette ou le Bailli dupé. Comedie en trois actes, et en prose, mêlée d'ariettes, Ec., Québec, J. Neilson, 1808, [6]-78 p.

[1802] ", La Barre du jour, vol. I, nos 3-5, juillet-décembre 1965, p. 117-141.

-, "Les Républicains français, ou La Soirée du Cabaret. Comédie en un acte... ", La Barre du jour, vol. V, n 25, été 1970, p. 64-68.

, Quelques poèmes et chansons..., Montréal, The Lawrence M. Lande Foundation, McGill University, 1970 [voir aussi infra John Hare, sur l'œuvre de Quesnel, ainsi que Yves Chartier].

_- "Lucas et Cécile", édité par Jean Marmier, Études canadiennes, $\mathrm{n}^{\circ} 16$, juin 1984, p.23-30.

ROUBAUD, Pierre-Joseph-Antoine, Lettres de Monsieur le Marquis de Montclam, gouverneur-general en canada, a Messieurs de Berryer $\mathcal{E}$ de la Molé écrites dans les années 1757; 1758, \& 1759: avec une version angloise, Londres, J. Almon, 1777, 28 p.

SALES LATERRIÈRE, Pierre de, Mémoires de Pierre de Sales Laterrière et de ses traverses, édition intime, Québec, Imprimerie de l'Événement, 1873, 271 p. (édition posthume).

SALES LATERRIÈRE, Pierre-Jean de, Journal de voyage [1815], [voir Andrès et Lespérance, 1994].

[pseudonyme A Canadian], A Political and Historical Account of Lower Canada; with Remarks in the Present Situation of the People as Regards Their Manners, Character, Religion, $\mathcal{E}$, $\mathcal{E}$, London, William Marsh And Alfred Miller, 1830, xvi-275 p.

SANGUINET, Simon, Le Témoin oculaire de la guerre des Bastonnais dans les années 1775-1776 [voir Verreau, 1873, p. 1-156].

\subsection{Anthologies/rééditions}

ANDRÈS, Bernard et Pascal RIENDEAU, La Conquête des lettres au Québec, 17661815, Montréal, Cahiers de l'ALAQ, n 1, UQAM, hiver 1993, 465 p.

HARE, John, "Journal du voyage de M. Saint-Luc de La Corne dans le navire l'Auguste en l'an 1761. Édition critique ", Revue d'bistoire littéraire du Québec et du Canada français, $\mathrm{n}^{\circ} 2,1980-1981$, p. 136-161.

LACROIX, Yvon-André, aUn Français et un Québécois dénoncent la Révolution française. Deux textes anciens de 1793 et 1799", Écrits du Canada français, $\mathrm{n}^{\circ} 30,1970$, p. 191-254. 
LAGRAVE, Jean-Paul de et Jacques G. RUELLAND (éd.), Pierre du Calvet. Appel à la justice de l'Etat $\varepsilon c$., extraits, Sainte-Foy, Le Griffon d'argile, 1986, 66 p.

LORTIE, Jeanne d'Arc (dir.), avec la collaboration de Pierre Savard et de Paul Wyczynski, Les Textes poétiques du Canada-français, 1606-1867, édition intégrale annotée, tome I : 1606-1806, 1987, xvii-613 p.; tome II : 1807-1826, 1989, xxiii-739 p.

MARCOTTE, Gilles (dir.), Anthologie de la littérature québécoise, vol. II : La Patrie littéraire, 1760-1895, sous la direction de René Dionne, Montréal, La Presse, 1978, xiv-516 p.

TÊTU, Henri et Charles-Octave GAGNON (dir.), Mandements, lettres pastorales et circulaires des évêques de Québec, tome II : 1741-1806, Québec, Côté et Cie, 1888, $566 \mathrm{p}$.

VERREAU, Hospice-Anthelme (dir.), Invasion du Canada. Collection de mémoires recueillis et annotés, Montréal, Eusèbe Sénécal, 1873, 394 p.

\section{OUVRAGES DE RÉFÉRENCE}

Ne sont ici mentionnés que les ouvrages renvoyant plus directement à la problématique du dossier.

ANDRÈS, Bernard, Écrire le Québec: de la contrainte à la contrariété. Essai sur la constitution des lettres, Montréal, XYZ éditeur, coll. "Études et documents", 1990,225 p.

, "Le texte embryonnaire ou l'émergence du littéraire au Québec: 17641815", Québec Studies, no 15, automne 1992-hiver 1993, p. 67-76.

—, «Du faux épistolaire: Pierre-Joseph-Antoine Roubaud et les Lettres de Monsieur le Marquis de Montcalm [...] écrites dans les années 1757, 1758, 1758 ", communication au colloque international La Lettre au xVIf siècle et ses avatars, 29 avril-1 ${ }^{\mathrm{er}}$ mai 1993, Collège universitaire Glendon, Université York, Toronto (à paraître aux Éditions du GREF).

(dir.), Principes du littéraire au Québec, 1766-1815, Montréal, Cahiers de l'ALAQ, nº 2, UQAM, été 1993, 65 p.

ANDRÈS, Bernard et Pierre LESPÉRANCE, Fortunes et infortunes d'un dandy canadien. Pierre-Jean de Sales Laterrière: Journal de voyage [1815], Montréal, Cahiers de l'ALAQ, no 3, UQAM, hiver 1994, 87 p.

ANGENOT, Marc, "Que peut la littérature? Sociocritique littéraire et critique du discours social ", La Politique du texte. Enjeux sociocritiques. Pour Claude Duchet, Lille, Presses de l'Université de Lille, 1992, p. 9-27.

BEAUDOIN, René, «Pierre de Sales Laterrière, médecin, mémorialiste et prototype de l'aventurier des lettres ", Bernard Andrès, Principes du littéraire au Québec, 1766-1815, op. cit., p. 43-56.

BRUNET, Manon, La Littérature française du Québec de 1764 à 1840. Essai pour une sémantique bistorique, thèse de doctorat en études françaises, Montréal, Université de Montréal, 1984, x-555 f, 2 vol. 
BRUNET, Michel, Les Canadiens après la Conquête, 1759-1775. De la révolution canadienne à la Révolution américaine, Montréal, Fides, 1969, 313 p.

BRYMNER, Douglas, "Rapport sur les archives historiques", Rapport sur les archives canadiennes, Ottawa, Roger Maclean et Cie, 1885, p. xiv-xxii.

BURGER, Beaudoin, L'Activité théâtrale au Québec, 1765-1825, Montréal, Parti pris, $1974,410 \mathrm{p}$.

CASGRAIN, Henri-Raymond, CEuvres complètes de l'abbé H. R. Casgrain, tome II : Biographies canadiennes, Montréal, Beauchemin et Valois, 1885, 542 p.

—, "Montcalm peint par lui-même d'après des pièces inédites ", Mémoires de la Société royale du Canada, section I, 1889, p. 3-27.

.__, "Le naufrage de L'Auguste", Bulletin des recherches bistoriques, juillet 1901, p. 207-209.

CHERRIER, Côme-Séraphin, L'Honorable F.-A. Quesnel, Montréal, J. Chapleau \& fils, imprimeurs et relieurs, $1878,26 \mathrm{p}$.

Dictionnaire biographique du Canada, tome II : de 1701 à 1740, 1969; tome III : de 1741 à 1770,1974 ; tome IV: de 1771 à 1800, 1980; tome V: de 1801 à 1820, 1983; tome VI: de 1821 à 1835, 1987; tome VII : de 1836 à 1850, 1988, Québec, Les Presses de l'Université Laval.

DIONNE, Narcisse-Eutrope, Inventaire cbronologique des livres, brochures, journaux et revues publiés en langue française dans la province de Québec, depuis l'établissement de l'imprimerie en Canada jusqu'à nos jours, 17641905, Québec, [s.é.], 1905, 175 p.

- Inventaire chronologique des livres, brochures, journaux et revues publiés en langue anglaise dans la province de Québec, depuis l'établissement de l'imprimerie en Canada jusqu'à nos jours, 1764-1906, tome III, Québec, [s.é.], 1907, viii-228 p.

, Inventaire chronologique des livres, brocbures, journaux et revues publiés en diverses langues dans et bors la province de Québec, $1^{\text {er }}$ supplément, 1904-1912, Québec, [s.é.], 1912, 76 p.

DOUCETTE, Leonard E., Theatre in French Canada. Laying the Foundations, 1606-1867, Toronto, University of Toronto Press, 1984, x-290 p.

DUMONT, Fernand, Genèse de la société québécoise, Montréal, Boréal, 1993, 393 p.

EMELINA, Jean, "La bourgeoisie dans la comédie de mœurs du xx $x^{e}$ siècle: exécration et exaltation ", Revue d'bistoire littéraire de la France, vol. LXXXIV, $\mathrm{n}^{\mathrm{O}} 3$, mai-juin 1984, p. 414-431.

FOUCAULT, Michel, L'Archéologie du savoir, Paris, Gallimard, coll. * Bibliothèque des sciences humaines ", 1969, 275 p.

- L'Ordre du discours. Leçon inaugurale au Collège de France prononcée le 2 décembre 1970, Paris, Gallimard, 1971, 81 p.

GAGNON, Serge, Le Québec et ses bistoriens de 1840 à 1920, La Nouvelle-France de Garneau à Groulx, Québec, Les Presses de l'université Laval, coll. "Les cahiers d'histoire de l'Université Laval n, $\mathrm{n}^{\circ} 23,1978,474 \mathrm{p}$. 
GALARNEAU, Claude, La France devant l'opinion canadienne, 1790-1815, préface d'André Latreille, Québec, Presses de l'Université Laval, coll. «Les Cahiers de l'Institut d'histoire ${ }^{n}, \mathrm{n}^{\circ} 16,1970, \mathrm{xi}-401 \mathrm{p}$.

GALLICHAN, Gilles, Livre et Politique au Bas-Canada, 1791-1849, Sillery, Septentrion, 1991, 519 p.

GOULEMOT, Jean-Marie et Daniel OSTER, Gens de lettres, écrivains et bohèmes: l'imaginaire littéraire, 1630-1900, [s.l.], Minerve, 1992, 199 p.

GRAFTON, Anthony, Faussaires et Critiques. Créativité et duplicité chez les érudits occidentaux, traduit de l'anglais par Murielle Cartier, Paris, Les Belles Lettres, coll. "Histoire ", 1993, 162 p.

GRENON, Michêl (dir.), L'Image de la Révolution française au Québec, 1789-1989, Montréal, Hurtubise HMH, coll. «Les Cahiers du Québec/Histoire n, 1989, $269 \mathrm{p}$.

GROULX, Lionel, Lendemains de conquête. Cours d'bistoire du Canada à l'Université de Montréal, 1919-1920, Montréal, Bibliothèque de l'Action française, 1920, 235 p.

GUMBRECHT, Hans Ulrich, "Le théâtre français du xviII ${ }^{\mathrm{e}}$ siècle comme véhicule des Lumières ", Diogène, n 136, octobre-décembre 1986, p. 103-127.

HABERMAS, Jürgen, L'Espace public. Archéologie de la publicité comme dimension constitutive de la société bourgeoise, Paris, Payot, 1978, 324 p.

HARE, John, "Quesnel et l'anglomanie de la classe seigneuriale au tournant du $\mathrm{xTX}^{\mathrm{e}}$ siècle ", Co-incidences, $\mathrm{n}^{\circ} 6,1976$, p. 23-31.

, La pensée socio-politique au Québec, 1784-1812. Analyse sémantique, Ottawa, Editions de l'Université d'Ottawa, 1977, $102^{\circ} \mathrm{p}$.

- Aux origines du parlementarisme québécois, 1791-1793, Sillery, Septentrion, coll. "Étude et documents", 1993, 305 p.

, Le Développement des partis politiques à l'assemblée législative du BasCanada, 1792-1814, Ottawa, Éditions Fontenay, 1994, 137 p.

HAYNE, David, "Le théâtre de Joseph Quesnel ", Archives des lettres canadiennes, tome V: Le Théatre canadien-français, Montréal, Fides, 1976, p. 109-117,

HUSTON, James [compilateur], Le Répertoire national: ou recueil de littérature canadienne, Montréal, De l'imprimerie de Lovell et Gibson, 1848, 4 vol.; deuxième édition, Montréal, J. M. Valois \& Cie, libraires-éditeurs, 1893, 4 vol.

JEANDILLOU, Jean-François, Supercheries littéraires. La vie et l'œuvre des auteurs supposés, Paris, Usher, 1989, xii-513p.

LAGRAVE, Jean-Paul de, Fleury.Mesplet (1734-1794): diffuseur des Lumières au Québec, Montréal, Patenaude éditeur, 1985, xv-503 p. Iréédité sous le titre L'Époque de Voltaire au Canada. Biographie politique de Fleury Mesplet, imprimeur, Montréal, L'Étincelle éditeur, 1993, 503 p.].

LAGRAVE, Jean-Paul, Jacques G. RUELLAND, Premier journaliste de langue française au Canada. Valentin Jautard, 1736-1787, préface de Jean-Louis Gagnon, Sainte-Foy, Le Griffon d'argile, 1989, 390 p. 
LAMONDE, André (dir.), L'Imprimé au Québec. Aspects bistoriques, $18^{e}-20^{e}$ siècles, Québec, Institut québécois de recherche sur la culture, 1983, 368 p.

LANCTÔT, Gustave, "Le prince des faussaires ", Faussaires et Faussetés en histoire canadienne, Montréal, Les Éditions Variétés, 1948, p. 171-201.

—_, "La vie scandaleuse d'un faussaire", Mémoires de la Société royale du Canada, Ottawa, Société royale du Canada, tome L, $3^{\mathrm{e}}$ série, juin 1956, première section, p. 25-48.

—, Le Canada et la Révolution américaine, 1774-1783, Montréal, Librairie Beauchemin, 1965, $330 \mathrm{p}$.

LATOUCHE, Daniel (dir.), avec la collaboration de Diane Poliquin-Bourassa, Le Manuel de la parole. Manifestes québécois, tome I: 1760 à 1899, Sillery, Boréal Express, 1977, 215 p.

LE MOINE, Roger, "Francs-maçons francophones de la "Province of Quebec" ", Cabiers des Dix, vol. XIVIII, 1993, p. 87-118.

LÉFIER, Yves, "Colas et Colinette ou le Bailli dupé et la réalité cànadienne ", Revue d'bistoire littéraire du Québec et du Canada français, $\mathrm{n}^{\circ} 12,1986$, p. 211-234.

LEMIRE, Maurice (dir.), La Vie littéraire au Québec, tome 1: La voix française des nouveaux sujets britanniques, 1764-1805, Sainte-Foy, Presses de l'Université Laval, 1991, 498 p., tome II : Le Projet national des Canadiens, 1806-1839, Sainte-Foy, Presses de l'Université Laval, 1992, 587 p.

- Formation de l'imaginaire littéraire au Québec, 1764-1867, Montréal, l'Hexagone, coll. «Essaịs littéraires ", 1993, 280 p.

LESPÉRANCE, Pierre, "Saint-Luc de La Corne et le naufrage de l'Auguste: la constitution d'un héros", Bernard Andrès (dir.), Principes du littéraire au Québec, 1766-1815, op. cit., p. 34-41.

- Saint-Luc de La Corne et le naufrage de l'Auguste : la constitution d'un béros, Montréal, mémoire de maîtrise en études littéraires, Montréal, Université du Québec à Montréal, 1994, 222 p.

MAHEUX, Arthur, "Notes sur Roubaud et sur sa responsabilité dans la nomination de $M$. Briand comme évêque de Québec", Société canadienne d'bistoire de l'Église catbolique, rapport 6, 1938-1939, p. 45-60.

MARION, Séraphin, Les Lettres canadiennes d'autrefois, tome II : La phase française, Ottawa/Hull, Éditions de l'Université d'Ottawa/Les Éditions "l'Éclair ", 2e édition, 1953, 191 p.

MASSEAU, Didier, L'Invention de l'intellectuel dans l'Europe du xVII siècle, Paris, Presses Universitaires de France, 1994, 172 p.

MCLACHLAN, Robert Wallace, "Fleury Mesplet, the First Printer at Montreal ", Mémoires de la société royale du Canada, $2^{\mathrm{e}}$ série, vol. XII, 1906, section II, p. 197-309.

_- "Some Unpublished Documents Relating to Fleury Mesplet ", Mémoires de la société royale du Canada, $3^{\mathrm{e}}$ série, vol. XIV, 1920, section II, p. 85-95. 
NOËL, Danièle, Les Questions de langue au Québec. 1759-1850. Étude revue pour le Conseil de la langue française, Québec, Éditeur officiel du Québec, 1990, xiv-397 p.

OUELLET, Fernand, "Nationalisme canadien-français et laïcisme au Xxx siècle", Jean-Paul Bernard (dir.), Les Idéologies québécoises au $19^{e}$ siècle, Montréal, Boréal-Express, 1973, p. 37-60.

- Le Bas-Canada, 1791-1840: changements structuraux et crise, Ottawa, Éditions de l'Université d'Ottawa, coll. "Cahiers d'histoire de l'Université d'Ottawa $" \mathrm{n}^{\circ}$ 6, 1976, $541 \mathrm{p}$.

PARIZEAU, Gérard, La Vie studieuse et obstinée de Denis-Benjamin Viger, Montréal, Fides, 1980, 330 p.

RAJOTTE, Pierre, "Les pratiques associatives et la constitution du champ de production littéraire au Québec, 1760-1867 ", Revue d'bistoire de l'Amérique française, vol. XLV, $\mathrm{n}^{\circ} 4$, p. 545-572.

ROBERT, Lucie, L'Institution du littéraire au Québec, Québec, Les Presses de l'Université Laval, 1989, 272 p.

ROCHE, Daniel, Les Républicains des lettres. Gens de culture et Lumières au XVII ${ }^{e}$ siècle, Paris, Fayard, 1988, 393 p.

ROCHEMONTEIX, Camille de, Les Jésuites et la Nouvelle-France au xVII siècle, d'après beaucoup de documents inédits, tome II, Paris, Picard, 1906, 305 p.

ROQUEBRUNE, Robert de, "Le naufrage de l'Auguste", L'Amérique française, 1953, p. 33-39.

—_, Les Canadiens d'autrefois, tome II, Montréal, Fides, 1966, 188 p.

ROY, Camille, Nos origines littéraires, Québec, Imprimerie de l'Action sociale, 1909, $354 \mathrm{p}$.

ROY, Pierre-Georges, $\grave{A}$ travers «Les Anciens Canadiens * de Pbilippe Aubert de Gaspé, Montréal, Ducharme, 1943, 279 p.

SAINT-JACQUES, Denis, "Fonctions et statut discursif de L'Appel à la justice de l'État ", Canadian Literature/Littérature canadienne, $\mathrm{n}^{\circ} 131$, hiver 1991, p. 64-71.

SERPILLON, François, Code du faux: ou commentaire sur l'ordonnance du mois de juillet 1737: avec des notes sur chaque article, une instruction pour les experts, en maitre de faux; plusieurs questions de droit concernant le crime de faux; \& un recueil des edits, arrets et reglements concernant les peines contre les fausaires, Lyon, G. Régnault, 1774, iv, 488 p.

SULTE, Benjamin, Mélanges historiques. Complétées, annotées et publiées par Gérard Malcbelosse, vol. VII, Montréal, G. Ducharme, 1921, 163 p.

TREMAINE, Marie, A Bibliography of Canadian Imprints, 1751-1800, Toronto, Les Presses de l'Université de Toronto, 1952, $705 \mathrm{p}$.

TRUDEL, Marcel, L'Influence de Voltaire au Canada, tome I: 1760 à 1850, Montréal, Fides/Les Publications de l'Université Laval, 1945, 221 p. 
VACHON, Auguste Georges, Pierre Roubaud: ses activités à Londres concernant les affaires canadiennes, 1764-1788, mémoire de maîtrise, Ottawa, Université d'Ottawa, Faculté des arts, Département d'histoire, 1973, xix-186 f.

WADE, Mason, Les Canadiens français de 1760 à nos jours, tome I: 1760-1914, traduit par Adrien Venne, avec le concours de Francis Dufau-Labeyrie, Ottawa, Le Cercle du livre de France, 1966, 685 p.

WALLOT, Jean-Pierre, Un Québec qui bougeait: trame socio-politique du Québec au tournant $d u X_{X} X^{e}$ siècle, Montréal, Boréal Express, 1973, 345 p.

WEINMANN, Heinz, Du Canada au Québec. Généalogie d'une bistoire, Montréal, l'Hexagone, 1987, 477 p. 